\title{
ERgonomía en la ENSEÑanza Clínica de la Estomatología
}

\author{
Guillermo Angulo Ocampo \\ Facultad Ciencias Estomatologia de la UIGV
}

\section{RESUMEN}

\begin{abstract}
En el presente trabajo se hace una revisión conceptual de los principios básicos de la aplicación de la ergonomía, que pensamos deben de considerarse en la formación clínica de los alumnos de estomatología. Planteamos un protocolo de atención clínica mediante la ejecución sistematizada de los procedimientos operatorios con una concepción nueva desde el punto de vista ergonómico satisfaciendo las diversas necesidades de la dinámica funcional de la atención del paciente y la calidad de servicio que pueden ser de utilidad en la aplicación de la ergonomía en Estomatología
\end{abstract}

Palabras clave: Ergonomía, procedimientos operatorios, sistema, comunicación.

\section{INTRODUCCION}

La Ergonomía (del griego Ergon = Trabajo y Nomos = Regla) inicia un nuevo sistema de adaptación del trabajo al hombre de acuerdo a sus modelos y métodos bajo una concepción del respeto del medio ambiente en el cual se desarrolló. La ergonomía, ciencia multidisciplinaría, participa en todos los procesos relacionados con la concepción, elaboración y uso del entorno habitable y laboral, adecuándolo al usuario.

Por eso, la ergonomía participa en el proceso de diseño e interviene en la creación del entorno artificial para el uso del hombre, teniendo presente su bienestar físico y mental.

La Ergonomía «no es un lujo, es esencial» tanto para el odontólogo como para los pacientes y el equipo de trabajo, así como para el ambiente de la clínica dental, el cual hoy día estamos modernizando, incorporando nuevas tecnologías $y$ materiales de manera constante. Ia ergonomía como tal, es una ciencia que no es de uso exclusivo en el campo de la odontología. Se puede afirmar que hastá ahora no se conoce desde cuando se aplica siendo tan antigua como la historia del ser humano.

En efecto, la necesidad de adaptar herramientas a la actividad del hombre, ha existido desde los tiempos más remotos y el avance de la civilización ha estado muy ligado a este propósito con la aplicación de la ciencia y la tecnología actual. Ante este estado de la ergonomía y hacia una interpretación coherente, observamos que el espacio del odontólogo debe ser rentable y optimizado al máximo de la clínica, según las funciones que van ha realizar en las diferentes áreas de la consulta. $\mathrm{Ha}$ sido nuestra preocupación desde hace mucho tiempo que las diversas funciones que se realizan en al clínica cuenten con el mayor número de ventajas, comodidad, ya que el esfuerzo que estas demandas originan una tensión psicológica muy grande.

Es por eso, la preocupación e interés que ésta disciplina tan importante e indispensable se aplique y se enseñe desde 
la formación del alumno de pre-grado para sentar las bases sólidas de una formación científica acompañada de los conceptos de calidad total en el servicio clínico.

\section{Aplicación de la Ergonomía}

Ante ésta problemática, surgen algunos planteamientos referentes a la aplicación concreta de la ergonomía en la enseñanza de la Clínica Estomatológica.

- Nivel de Pre-Grado Trabajo clínico a 4 manos siguiendo un protocolo de aprendizaje y atención clínica en los pacientes, bajo normas de bioseguridad.

- Nivel Profesional Ergonómico: aplicación de los conocimientos de ergonomía para lograr el mayor rendimiento, calidad y eficiencia en el desarrollo del personal auxiliar, se reduce el nivel de estrés y aumenta la eficiencia de la atención para beneficio de los pacientes y el personal.

Para lograr este objetivo, se deben tener en cuenta los siguientes niveles ergonómicos:

1. El personal odontológico, auxiliares, asistentas, secretarias y personal de servicio a higiene deben trabajar bajo normas de ergonomía y bioseguridad en la clínica estomatológica.

2. Elaboración de diseño y planificación de los espacios físico, tránsito, ventilación, iluminación, sonido, contaminación y funcionalidad ergonómica de los espacios.

3. Selección y manejo del instrumental, materiales y equipos mediante el trabajo asistido a 4 manos.

4. Posición ergonómica correcta del operador y asistenta durante el trabajo clínico.
5. Planificación y simplificación de técnicas y procedimientos administrativos entre los pasos clínicos y de laboratorio.

6. Uso de un soporte informático.

7. Confort del espacio físico de trabajo, con un buen nivel de comunicación.

Menciono alguno de los aspectos más significativos de la aplicación de la ergonomía que tendrá como resultado la disminución de las cargas físicas y psíquicas creando un clima de armonía y alto rendimiento. $Y$ el reto será poner en práctica un «sistema de atención ergonómica», que sería una revolución completa de la calidad de enseñanza clínica de la estomatología, la evolución de tecnologías de la información en los albores de la era digital y de las redes (Internet) introduce modificaciones profundas en las posibilidades del acceso al saber y de su difusión como el proceso enseñanza - aprendizaje.

\section{CONCLUSIÓN}

1. Mediante la aplicación de la ergonomía. prevenimos los riesgos profesionales, mejora de las condiciones de trabajo (confort) mejora de la calidad de servicio, fiabilidad y mayor cobertura de atención, siendo estos aspectos estratégicos para una formación integral del estudiante de la clínica.

2. Se debe investigar respecto a la implementación de un sistema de atención ergonómica fijando como meta la optimización estratégica de servicio, rendimiento y calidad de servicio.

3. La «practica asociada» se ve ampliamente beneficiada cuando se ejerce tomando en consideración la ciencia de la Ergonomía. 


\section{REFERENCIAS}

1. Calatayud, Sierra J. Diseño de la Consulta Dental. Cap. XIV. Ergonomía y Control de la infección en odontología, 1997.

2. Carlos Hibas, Juan. Ergonomía - ISAT CEPIS.htm.2001.

3. Cazamin P., Ergonomía: El Ejercicio de la Medicina del Trabajo. Ed. Científica Médica, Barcelona, 1990.

4. http://www.educnet.education.fr/esp/ formation/competencias
5. http:www.odontomarketing.bizland.com Revista: Virtual de la Práctica Odontológica. N. 13. Mayo 2001.

6. Ins@sinfo.net \{mailto:jbs@sinfonet [Juan San Martín.100 ideas de mercadeo. 1999]\}

7. Hosepn Echantena. 2a ${ }^{a}$ Edición, 1994. Barcelona.

8. Mundo Odontológico. Revista Anual V.No. 16. 1999. Lima - Perú.

9. URL.: A:/Ergonomía Digital.htm. Ergonomía Digital 2001.

10. Wisner A.: Ergonomía en Odontología. Ed. Jims. Barcelona. 1991. 